\title{
Computer-based learning in psychology using interactive laboratories
}

\author{
Stephen Richards
}

\author{
Department of Psychology, University of Newcastle-upon-Tyne
}

\begin{abstract}
Traditional approaches to computer-based learning often focus on the delivery of information. Such applications usually provide large stores of information which can be accessed in a wide variety of ways. Typical access facilities provided within such applications include Boolean search engines and hypermedia (non-linear) browsing. These types of approach often centre on providing human-computer dialogues which are relatively low on interaction. The interactive-laboratory approach, however, aims to limit the quantity of information presented, and instead to provide a highly interactive learning environment. In the field of psychology, users are able interactively to design and deliver a broad range of psychological experiments. This paper details the approach, and describes how it can be used to teach psychology within a university environment. The way in which its effectiveness as a learning tool can be evaluated is also discussed.
\end{abstract}

\section{Introduction}

Educational technology is currently receiving a great deal of attention and resource, particularly in higher education. The reason for this is that higher-education establishments are being required to deliver ever-increasing numbers of graduates on evertightening budgets. A number of approaches can be taken to counter the problems associated with this situation. Some institutions are building ever-bigger lecture theatres to allow greater numbers of students to access the expertise of the teacher. But this approach tends to produce passive learning experiences when compared to the interactive learning which often takes place when only small numbers of students are addressed, and although such lectures are useful, within many subject domains the transmission of appropriate procedural, experimental and practical skills is essential to useful learning experiences. Another approach, as readers of this journal will know, centres on the use of computer technology for the dissemination of both skills and knowledge, since computer-based learning applications can provide an interactive medium for learning experiences. Many traditional paper-based learning-support materials are essentially static and unreactive: 
information is usually presented in a predetermined way with a fixed set of pedagogical objectives. Although users are often able to override such objectives, interaction with traditional learning materials is limited (see Richards, 1993), while interactive computerbased learning environments are able to respond to the individual requirements of users from information elicited through the interaction process. In this way, a learner-centred approach can be adopted. Although this does not preclude teacher-centred approaches, it nevertheless allows individual users to tailor their learning experiences.

\section{Related work}

A number of programs have been written which make use of an experimental laboratory approach to the teaching of psychology (see, for example, Chute, 1989; Tversky, 1990; see also Cox et al., 1992). Several programs of this kind are reviewed in detail in Psychology Software News, for instance Laboratory in Cognition and Perception (Smith, 1990). This typical application allows users to run experiments in a number of areas within the general field of cognition and perception, and when the program is operating in advanced mode, users are able to set a number of parameters prior to running an experiment. Results are stored in files which can then be analysed fully at a later date. But this application was designed for relatively primitive computing technology by today's standards, as shown by the fact that although a colour monitor is recommended, all the experiments are designed so that they can be delivered in monochrome. Today's technology allows far more ambitious uses of a laboratory approach. In particular, multimedia provides a much broader scope for both the quality and the range of experimental work that can be supported. In any case, the use of multimedia aside, such applications differ from the genuine interactive laboratory in the following ways:

- they do not employ multiple pedagogic strategies;

- student remediation strategies are usually not embedded;

- neither existing laboratory materials nor experiment generators are integrated.

\section{Objectives}

The principle objective in this area of my research, therefore, is to create highly interactive learning materials which allow students to become actively engaged in the setting up and delivery of psychological experiments. These experiments are to be controlled through the use of graphical user-interfaces which will allow relatively naive computer users rapidly to develop, deliver and analyse data for a wide range of psychological theories and concepts, the whole being enhanced through the use of multimedia technologies. In addition, appropriate existing software can be integrated into the system. This requires copyright clearance from authors and, of course, some changes may need to be made to such applications to allow their integration into the interactive laboratory, which means obtaining permission to make alterations to the source code. The resulting learning materials are being developed and integrated in close consultation with academic staff engaged in teaching psychology at Newcastle University.

Of course, a major factor in the success of such software is the degree to which students use, enjoy and learn from the approach that has been adopted. Simply providing stimulating and interactive learning environments does not guarantee success. Methods 
are required to monitor student take-up, continued use, and attitudes towards the materials and strategies used for its delivery. For these reasons, it is also the objective of my current research to develop extensions to the system for monitoring who uses it, when they use it and for how long.

\section{Development environment}

A wide range of environments can be adopted for the development of interactive laboratories. There are, however, a number of features which are potentially extremely important, and which I now outline, as well as the way in which the adopted environment addresses the problems of delivering the necessary facilities.

\section{Hardware}

The use of interactive computer-based learning materials in an educational environment represents a delicate balance between cost and power. Fortunately, the rapid reduction in the cost/power differentials of desktop computers has meant that appropriate technologies are now widely available. In the Psychology Department at the University of Newcastle, there has been a move towards the use of Macintosh networks. The principal student machine, therefore, will be based on new Macintosh LC475s which have already been installed in one of the laboratories. These are networked (along with the other Macintosh machines in the department) using Ethernet and AppleTalk.

The development machine (which will later act as a file server) is a Macintosh Centris $660 \mathrm{AV}$. This machine has a number of advantages for the development of the interactive laboratory which stem from its in-built audio-visual capability, including integral audiol video digital signal processing hardware and a multi-session double-spin CD-ROM drive.

Apple Macintosh machines have long been equipped with audio digital signal processing hardware as standard, something which has given Apple an advantage over IBMcompatible hardware in the move towards multimedia. The audio circuitry is capable of producing 16-bit stereo audio with sampling rates of $22.05,24,44.1$ and $48 \mathrm{kHz}$. The Centris $660 \mathrm{AV}$, however, has the additional capacity to take in analogue video signals and produce digital video files for delivery from hard disk. North American, UK, and European video standards (NTSC, PAL and SECAM) are all supported. The video source (which may be a camcorder, video cassette recorder, or videodisk) can be linked through either the composite video or the SuperVHS port. Film clips can then be recorded to the hard disk in QuickTime digital video format. If the audio channel is also connected, the audio stream is interleaved to form a digital audio/video file. Such files can then be delivered in software alone on machines which do not possess the specialized video hardware. As for CD-ROM, the multi-session capability of the drive in the Centris 660AV means that CD-ROM discs can be written to more than once. This is a problem for some drives as they are only able to read discs which have been written in a single session, but such multi-session compatibility is essential for handling Kodak PhotoCD discs. It is possible to create a PhotoCD disc, then have additional images added at a later date (hence the term multi-session).

\section{Authoring}

Most of my development work so far for the interactive laboratory has been based 
around HyperCard 2.19, though this has recently been upgraded to 2.2. This new release provides significant increases in both power and flexibility, and in particular offers support for colour and multimedia. Colour was previously available to developers of HyperCard stacks only through the use of third-party executable commands and functions. Such facilities have now been included with HyperCard in the form of the ColorTools extensions which allow PICT format graphics files to be imported and resized. In addition, ColorTools allows the colouring of HyperCard objects such as buttons, fields, cards, and backgrounds. Multimedia support is provided through the integration of QuickTime and ADDmotion tools. ADDmotion provides 24-bit colour paint, animation and sound extensions. The QuickTime extensions allow interleaved digital video/audio files to be controlled from within HyperCard. A wide variety of window types and control options can now be simply embedded within an application using the toolkit provided. This means that video, animation and sound can now be controlled (with relative ease) from within HyperCard.

\section{Experiment generators}

It is envisaged that the use of two experiment generators (MindLab and SuperLab) will be integrated into the interactive laboratories. The modules that have been developed to date, however, have made use only of SuperLab (SuperLab, 1991) which allows psychological experiments to be set up and delivered on a computer. Both visual and auditory stimuli can be presented, and reaction times can be recorded accurately through a wide selection of input devices.

One of the features of SuperLab that makes it particularly useful in this type of work is the file formats it produces. Two basic types of file are used: script and output. The script file provides all the information SuperLab needs in order for it to run an experiment, while the output file stores a record of the stimuli presented, user-responses and reaction times. Both these files are tab-delimited text files. This means that a SuperLab-compatible script file can be generated within other environments, then SuperLab invoked with the generated script file. In addition, although the output file is a Microsoft Excel file, it simply consists of tab-delimited text and can therefore be imported into a wide range of applications.

\section{Design, monitoring and pedagogic strategy}

Although an interactive-laboratory approach can be used to teach a variety of scientific subjects, my current research is limited to teaching psychology. The following section therefore describes the general design and implementation of an interactive laboratory for psychology.

\section{Interface design}

One of the key problems with developing materials for teaching non-computing disciplines can be the relative lack of computing skills exhibited by some of the potential users. This is a particular problem with first-year students who may have little or no computing experience. Naturally, as part of any modern degree course in psychology, the use of computer resources has become a necessity, something which can result in a substantial load for non-computer-literate students. I have therefore adopted a highly 
graphical approach to user-interface design. The two principle interaction methods within any graphical user-interface are menus and icons. Although the interactive laboratory makes use of both these strategies simultaneously, all essential navigation controls are provided iconically at the base of the screen. This allows the completely naive user to be able rapidly to access the essential set of sub-functions without a significant learning curve. A greater range and subtlety of interaction is available through the menus for users who are more experienced.

\section{User monitoring}

The two most common methods used to address the problem of additional assistance for students experiencing learning problems are testing and interaction monitoring. Testing can be effective when students obtain immediate feedback on their performance on computer-based tests embedded in the learning environment. The object of this strategy is that it allows students to effect their own remedial action. But associated problems include de-motivation through poor attainment, lack of constructive personal feedback, and, in some cases, an inability to take appropriate remedial action. For these reasons, I have opted for interaction monitoring. This can take many forms, ranging from recording every interaction that the user makes within an application, to keeping a simple record of the length of time spent using a particular part of the application. Where all interactions are recorded, a very large amount of information is generated which in itself can obscure the identification of those students who may require remedial action. Thus, in the interactive laboratory, a time-monitoring strategy using student $\log$ files has been adopted, implemented in such a way that when a teacher accesses student-monitoring

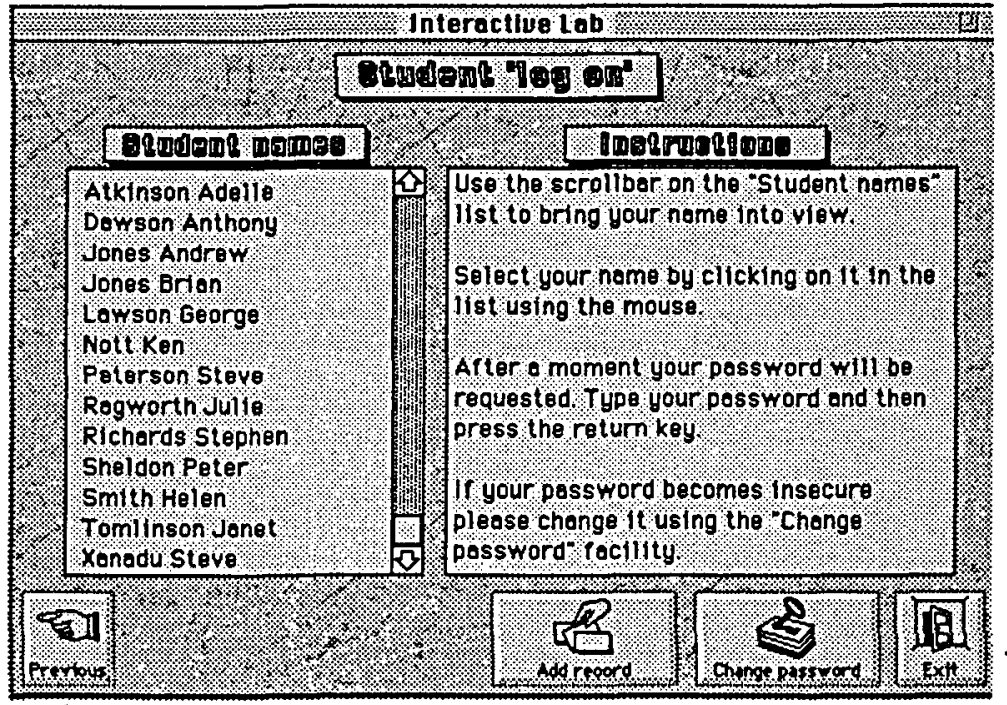

Figure 1: The student logging protocol used for interaction monitoring

information, he or she is able to identify the modules that have been accessed, by whom, when, and for how long. This means that students who either fail to access a particular module, or who take a disproportionately long time to complete a particular module, can be easily identified. (Such information is naturally held in protected files to prevent unauthorized viewing.) 


\section{Pedagogic strategles}

Three basic pedagogic strategies are implemented within the interactive laboratory. These involve the delivery of theoretical background information, the provision of instruction for the various software tools, and interactive control of practical sessions.

The delivery of background information is based on a simple presentation. This involves several screens of information outlining the key features of research conducted in the area, and any details of how current methodology differs from the original work. However, in order to avoid long periods of reading from screen, information is kept to a minimum and relies on references to further reading.

At present, the training approach to the use of software tools supports only a brief introduction to SuperLab. Users are provided with active learning facilities which allow them to toggle between SuperLab and the instructional materials, thus providing realtime training. In this way, they are led through the process of running experiments interactively within a SuperLab session. Through the use of this technique, even students who have no knowledge of SuperLab, and who are also low in computing experience, are able successfully to run experiments from within the SuperLab environment.

The educational approach to the laboratory sessions is based on an active learning strategy. Naturally, the specific design of each laboratory session is specific to an individual module, since the particular features of each experiment have a determining effect on the facilities and methods employed in the user-interface. Even so, the basic strategy is to allow users to set up a wide range of experimental parameters. In this way, students can design their own experiment which they are then able to deliver through the computer. Results data is automatically collected into a file of their choice for later statistical analysis.

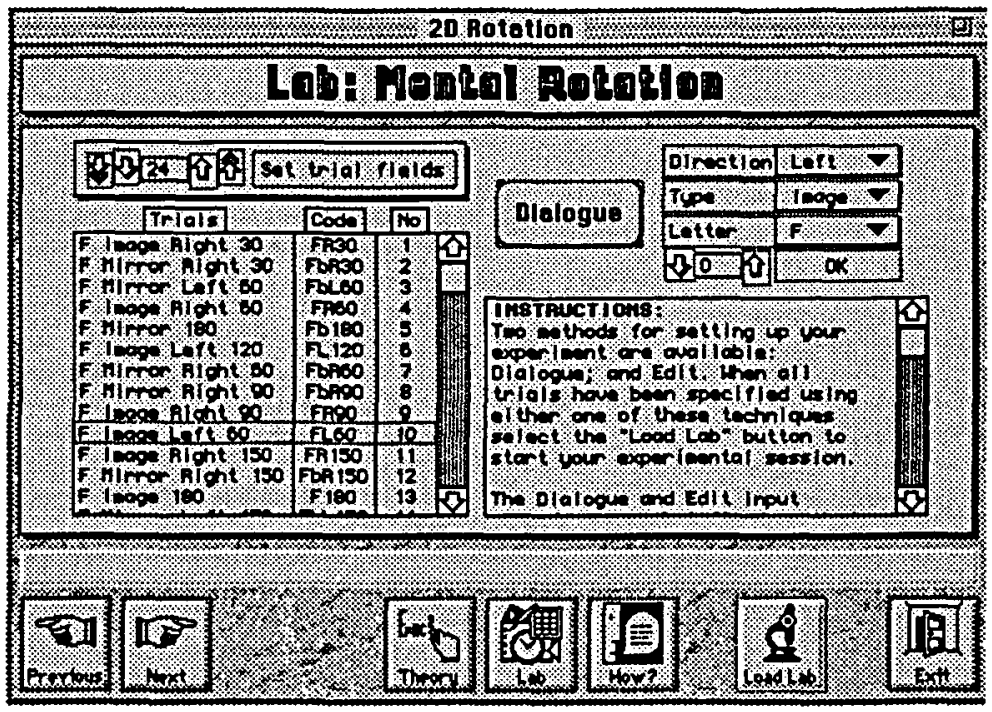

Figure 2: The graphical user-interface used in setting up experiments

\section{Case studies}

Two techniques are used in the creation of laboratory sessions: experiment generators and direct coding in HyperCard. The experiment-generator approach involves the creation of 
interfaces which allow the necessary parameters to be written to a SuperLab script. SuperLab is then invoked to deliver the stimuli and collect the data. The direct coding approach can involve the modification and use of existing software and/or the complete coding of new modules in HyperCard. In the following outline case studies, both of these approaches are illustrated.

\section{Mental rotation}

A laboratory session has been produced which addresses the issues of mental rotation following the work of Shepard and Metzler (1971). This experiment measures the time taken (in milliseconds) to rotate an image or a mirror image mentally to its original orientation. For the purposes of this experiment, SuperLab was integrated to deliver the experimental stimuli and collect the resulting data. This was achieved by launching the run-time version of SuperLab with a generated SuperLab script file from within a HyperCard stack.

Within the interactive laboratory, SuperLab script files are created from the parameters set by students. This is achieved through selecting the parameters required for each event within a particular experiment using a graphical user-interface. Once the parameters for all events have been set, they are then passed to SuperLab via the script file. At present, the interactive laboratory supports up to 200 events within a single experimental administration.

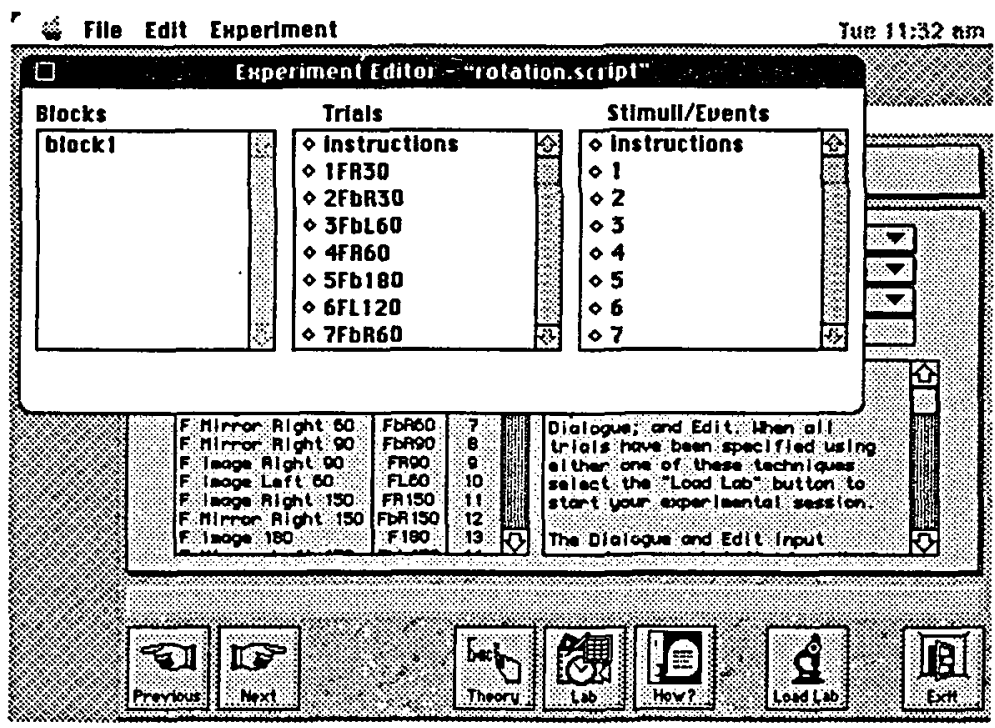

Figure 3: Users can toggle between SuperLab and the interactive laboratory in order to make modifications to the experiment or access instructional materials

\section{Short-term memory}

A different approach from that used for the mental rotation module has been used for the module dealing with short-term memory. This involved making use of an existing set of HyperCard stacks which were developed for the delivery of words and nonsense words using digital audio techniques. A full description of the original work can be found in Cox, Hulme and Brown (1992). In order to integrate this set of stacks, it was necessary to supplement the original code so that students could be provided with a brief introduction 
to the background theory, and online instruction on setting up and running experiments using the module. It was also necessary to embed the code for logging student interaction.

\section{Future directions}

Although I have discussed the monitoring of student interaction in the context of remediation, such monitoring can of course also be used in the wider context of software evaluation. This provides one of the key issues to be addressed in the near future as the modules go online in the teaching of psychology. In addition, although the use of text, graphics and digital audio has been mentioned above, the full potential of multimedia has not been addressed.

\section{Multimedia}

The strict definition of multimedia is the use of a number of presentation modalities within a computing environment, but it is sometimes - indeed often - taken to mean the use of digital motion video. Until very recently the use of digital video was almost prohibitively complex. More recently, however, improvements in hardware and software tools have meant that it can now be usefully employed in a wide range of applications (Richards and Barker, 1994).

Within the interactive laboratory, a number of uses for digital video are being investigated. Areas currently under investigation include eye-witness testimony and animal behaviour experiments.

In the eye-witness testimony work, QuickTime movies will be used to deliver digital-video scenarios to subjects from a scenario library held on CD-ROM. Students will be able to select a variety of approaches to gathering eye-witness testimonies via the computer which may include multiple-choice, free-flow response, structured response, graphical representations of evidence, and simulated identity parades.

It is envisaged that animal and human-behaviour experiments will be presented to students through the creation of an integrated behaviour-recording environment. Effectively, field studies will be simulated by presenting digital video clips from a resource library, and students will be able to specify the behaviours to be recorded along with the associated codes and key responses. Behaviour and timing information can then be recorded by the student while viewing the selected digital video clip.

\section{Evaluation}

Formal assessment of the impact of the approach described in this paper to teaching psychology will be conducted over the coming year. In addition to measuring the overall impact of the software, attempts will be made to identify the types of student who can benefit from such an approach, and perhaps more importantly, the types of student for whom such an approach may be inappropriate.

A number of tools are under development to support this evaluation process. As already mentioned, the logging of student interaction is already in place and it is expected that this will yield useful data. In addition, questionnaires are being formulated which will address the use of such methods from both educationist and student perspectives. 


\section{Conclusion}

The work described in this paper illustrates the way in which a number of pedagogic approaches can be implemented using computer-based learning materials. These include both relatively passive information presentation and highly active learning strategies in the form of interactive experiments and real-time training. In addition, the work illustrates ways in which computers can be used to augment traditional approaches to teaching psychology. In many instances, traditional teaching methods can no longer support the extremely large numbers of students now faced by the modern lecturer undertaking practical work. The interactive-laboratory approach can facilitate access to active learning experiences.

Finally, although the focus of the work described here has been within the field of psychology, it is clearly possible to apply the same techniques within a variety of disciplines. Multimedia could have a particularly large impact in this regard, allowing the creation of life-like simulations which it otherwise may be difficult or impossible for students to access.

\section{Acknowledgements}

I acknowledge the University of Newcastle for funding through the Teaching Initiatives

Fund; Charles Hulme for permission to integrate the Short-Term Memory Experimenter which has been used in one of the modules; and both academic and technical staff in the Department of Psychology at Newcastle who have provided information, support, suggestions and criticism.

\section{References}

Chute, D. (1989), MacLaboratory Psychology Version 1.0 (computer program), Devon, PA, USA.

Cox, R., Hulme, C. and Brown, G.D.A. (1992), 'STM Experimenter: using HyperCard and MacRecorder in short-term memory experiments', Behaviour Research Methods, Instruments, and Computers, 24, 4, 575-9.

Richards, S.M. (1993), End-user Interfaces to Electronic Books, Ph.D. thesis, University of Teesside.

Richards, S.M. and Barker, P.G. (1994), 'A multimedia trip to MIT', Proceedings of the 11th International Conference on Technology and Education, London, in press.

Shepard, R.N. and Metzler, J. (1971), 'The mental rotation of three-dimensional objects', Science, 171, 701-3.

Smith, P.T. (1990), review of Laboratory in Cognition and Perception in Hammond, N. and Trapp, A. (eds), Psychology Software News, 1, 3, University of York, CTI Centre for Psychology, 1990, 52-4.

SuperLab (1991) (computer program), version 1.4, Silver Spring, MD, USA, Cedrus Corporation.

Tversky, B. (1990), Experiments in Cognitive Psychology (computer program), Stanford University Software Distribution Center, Stanford, CA, USA. 